Historic, Archive Document

Do not assume content reflects current scientific knowledge, policies, or practices. 



\section{$C 2.21$}
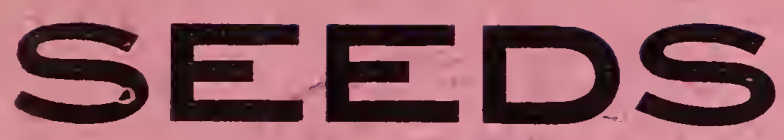

\section{8}

-Bulk Seed

Price List

\section{Garden,Flower,Field 画Grass Seed}

Bulhs, Plants, Nurrsery Stock and Garden Tools.

Clover, Alfalfa, Timothy,

Blue Grass, Seed Corn.

\section{MICHAEL'S Seed Store}

316 PEARL STREET,

J. S. MICHAEL, Prop.

SIOUX CITY, IOWA.

N. B. -We are always in the market to buy all kinds of Field and Grass Seeds if you have any to sell. Mail samples. 


\section{Pearl St.}

\section{Market Gardeners Attention.}

WISH to say to you that we handle nothing which we do not know to be good and the very best obtainable, and while we do not claim to compete with the trash that haphazard growers often sell to dealers, we will make you prices on a class of seeds true to name, pure and good test that will save you money by coming here to buy your seeds instead of sending a thousand miles away for them.

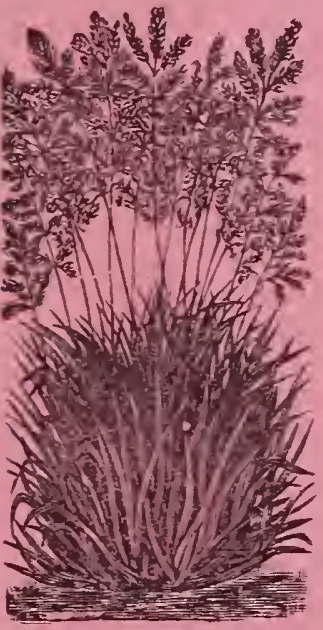

hentucky Blue Grass.

It is my aim and ambition to build up as reliable a retail seed store here in Sioux City for your benefit as can be found anywhere in the world. I know you will appreciate it and share your trade with me that, that result may be accomplished.

\section{SPECIAL OFFER,}

\section{5c papers 2 for a nickle.}

\section{2 for $25 \mathrm{C}$ \\ 25 for $50 c$.}

\section{Farmers Welcome}

You will make a mistake if you don't go to a seed store to buy your seed for both the field and the garden. I will spread your dollars thin and save you money every time.. Come and see me. You are always welcome. If you have any field seed to sell send me samples Millet, Clover, Timothy, etc. -

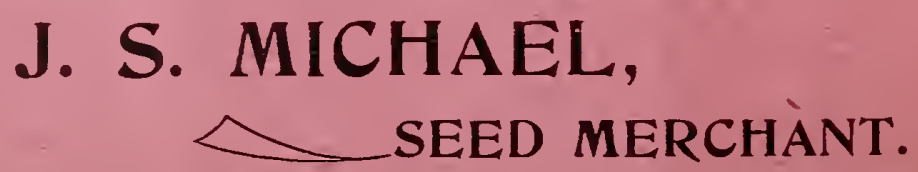




\section{Michael's Seed Store,}

\section{Pearl Street, SIOUX CITY, IOWA.}

REMEMBER to come and see us or send your orders by mail.

\section{GARDNER'S PRICE LIST BULK SEEES.}

We sell 5c papers two for a nickel.

CASH PRICES.

Artichokes-Green Globe Seed

LB. $B U$

Jerusalem Tubers

$\$ 125$

Asparagus-Seed, Barrs Mammoth

Seed, Columbian Mammoth White

Plants, 1 year roots

per 1,000

$\$ \quad \begin{aligned} & \text { Oz. } \\ & 25\end{aligned}$

"“ 2 year roots

"

Beans-Dwari Wax Sorts.

Golden Wax

PT. QT. PK.

German Black Wax $\$$

Detroit Wax

Davis Wax (Ferry's Stock)

$\begin{array}{rrr} & 5 & 30 \\ & 10 & 75 \\ 5 & 00 & \\ 600 & -\end{array}$

Ferry's Challenge Black Wax

$10 \$ 15 \$ 75$

$10 \quad 15 \quad 75$

$10 \quad 15 \quad 75$

$10 \quad 15 \quad 100$

White Wax

$10 \quad 15 \quad 80$

Wardwell's Kidney Wax

$10 \quad 15 \quad 80$

Flageolet Wax

$10 \quad 15 \quad 75$

$\begin{array}{lll}10 & 15 & 75\end{array}$

New Refugee Wax

$15 \quad 25 \quad 100$

Saddle Back Wax

$10 \quad 15 \quad 75$

Green Pod Sorts-Dwarf.

First in Market (Landreth's stock) -...- - - - - - $15 \quad 1525$

$10 \quad 15 \quad 75$

Early Six Weeks (round pod) _............. $10 \quad 15 \quad 75$

White Valentine =....................... $10 \quad 15 \quad 75$

Best of All .............

Extra Early Refugee (earliest of all) _........ $\quad 10 \quad 15 \quad 75$

Dwarf Horticultural

$15 \quad 25 \quad 125$

lmproved Goddard

$10 \quad 15 \quad 125$ 
Dwarf Lima Sorts.

PT. QT. PK.

Henderson's Bush Lima $\$ 10 \$ 15 \$ 100$

Burpee

$15 \quad 25 \quad 150$

Jackson's Wonder

$15 \quad 25 \quad 150$

Pole Varieties.

Large Lima, Dreer's Improved. ............ $\quad 15 \quad 25 \quad 135$

Lazy Wife ............................ $15 \quad 25 \quad 150$

Kentucky Wonder (Old Homestead) ......... $\quad 15 \quad 25 \quad 150$

Dutch Case Knife.................... $15 \quad 25 \quad 125$

Horticultural Pole........................ $10 \quad 1590$

Tall Sioux or Hemisphere ............... $10 \quad 15 \quad 75$

Cut Short or Corn Hill _... _... _...

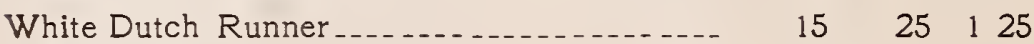

Field Sorts. $\quad$ PT. QT. PK. BU.

White Navy (home grown) ........... \$ $5 \$ 10 \$ 50$

Prolific Tree Bean................. $10 \quad 15 \quad 50$

White Marrow ..................... $5 \quad 10 \quad 40 \$ 125$

If to go by mail add $8 \mathrm{c}$ per pint.

Beets-Table Sorts.

OZ. LB.

Egyptian Extra Early Turnip Blood ............... \$ 5 \$ 40

Eclipse (Landreth's or Ferry's Stock) _........... 55

“ lmported Stock

Early Turnip Blood ............................ 5

Early Flat Red Bassano ........................ 55

Landreth's Early Forcing ........................ 55

Long Smooth Blood Red...................... 535

Ferry's Half Long Blood Red...................... 540

Sugar Sorts.

Imperial French Sugar

Red Top White Sugar _...

Klein Wanzleben

For Cattle.

Mammoth Long Mangle ....................... 525

Golden Tankard .............................. 5

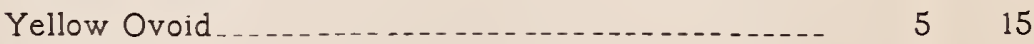

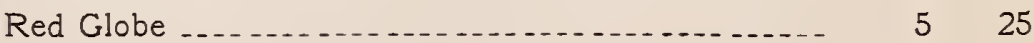

Brocoli-Large, Early White ........ 35

Landreth's Purple

Brussel Sprouts-Dwarf lmproved.................. 10

Cabbage-Early York (imported stock) _................. $10 \quad 75$

Landreth's Select Early Jersey Wakefield_......... 15200

Early Jersey Wakefield (imported stock) ............ $\quad 10 \quad 100$ 
Reedland,s Early Drumhead (Landreth's) .......... \$ 25

Extra Early Express (imported) ................ $10 \$ 100$

Early Winningstadt _................................ 10125

Henderson's Early Summer..................... 20

." " “ “ (Puget Sound) ....... 10125

Bloomsdale Early Market (Landreth's) ............ 20

.. " Drumhead “

Succession (Landreth's) $\ldots \ldots \ldots$

All Seasons ."

All Head Early “" _.................... 20

Early Flat Dutch (Puget Sound) _................... 10125

Market Gardeners Late Flat Dutch (Landreth's) _..... 20150

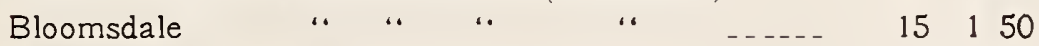

Late Flat Dutch (Puget Sound) _................... 10100

Sure Head " " "

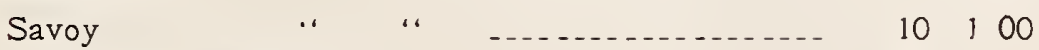

Red Dutch (Landreth's) ......................... 20

Carrots_Extra Early Forcing (Landreth's)_........... 10100

NEW HALF LONG CORELESS (Landreth's . ...... 50

OXHEART _...................................... 560

Danvers Half Long............................. 50

Long Orange ................................... 50

White or Yellow Belgium, for stock _............. 55

Cauliflower-Henderson's Snowball_................. 350

Ferry's Snowball ......................... 350

- Common Snowball ...

Extra Early Dwarf Erfert....................... 300

Celery-Giant Pascal (Landreth's) _................... 10

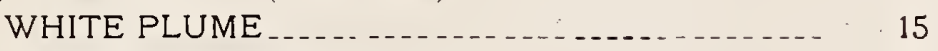

Golden Self Blanching ....................... 25

Celeriac, Turnip Rooted Celery .................. 10

Soup Celery ................................. 5

Chervil-Double Curled _.......................... 10

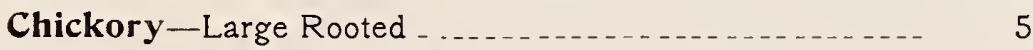

Collard's Georgia $\ldots \ldots \ldots \ldots \ldots \ldots \ldots$

Cress-Garden _...................................... 5

True Water Cress ............................ 25

Upland Cress................................. 25 
Sweet Corn-Early Cory

White Cob Cory

FERRY'S MAMMOTH WHITE COB CORY --

Early Minnesota ........................

Crosby's Early ........

Country Gentleman .........................

Black Mexican .... . . . . . . . . . . . . .

STOWELL'S EVERGREEN (select stock) -..-

Mixed Evergreen (for fodder) .... per bu. $\$ 125$

Mammoth Sugar.

If to go by Mail add $8 \mathrm{c}$ per pint.

PT. QT. PK.

$\$ 10 \$ 15 \$ 40$

$\begin{array}{lll}10 & 15 & 40\end{array}$

$15 \quad 25 \quad 160$

$10 \quad 15 \quad 50$

$10 \quad 15 \quad 40$

$10 \quad 15 \quad 50$

$10 \quad 15 \quad 40$

$10 \quad 15 \quad 50$

35

$15 \quad 75$

Oz. LB. $\$ 15 \$ 40$

540

540

540

540

Long Green

Egg Plant_Large Round Purple ........... 25

New York lmproved.......................... 25

Endive-Moss Curled . . . . . . . . . . . . . .

Broad Leaved Batavian ...................... $15 \quad 125$

Kale or Borecole-Tall Green Curled............... 56

Dwarf Curled .................................. 56

Kohl Rabi-Early White Vienna .................. 15

“ Purple "“ ............. 15

Leek-London Flag . . . . . .

Lettuce-GRAND RAPIDS. . . . . . . . . . . . . . . . . . $10 \quad 100$

Ferry's Early Prize Head .....

Simpson's Early Curled White Seeded ............ $\quad 1075$

Hanson

Tennis Ball .................................. 50

VIRGINIA SOLID HEADED . . . . _ . _......... $15 \quad 150$

Bloomsdale Butter .......................... 10125

CELERY LETTUCE ......................... 10150

Musk Melon-Jenny Lind ... . . . . . . . . _............... 55

Landreth's Early Green Citron Cantaloup ........... 55

SALMON \& GREEN (Landreth's) . ... ........... 55

Emerald Gem ............................ 540 
Extra Early Hackensack 0 OZ. LB. OSAGE.

Shumway's Giant............................. 540

Jersey Belle.................................. 55

ORANGE AND GREEN (my novelty) _......... 15

Water Melon_LANDRETH'SBOSS_._._._....... 5

Boss Cory's Stock. . . . . . . .

Ferry's Peerless _.............................. 55

Phinney's Early _............................... 50

Stokes Extra Early _........................... 5

Hungarian Honey ................................ 5

Cuban Queen ..................................... 535

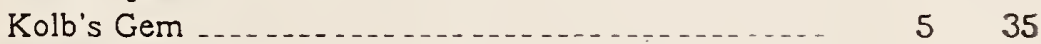

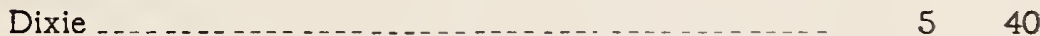

Mountain Sweet ............................... 540

Sweet Heart ................................. 540

Mushroom Spawn-English

French

Mustard-White London _....................... 1040

Okra-White Velvet ............................... 10

Onions_Extra Early Bloomsdale Pearl ............... 20

Extra Early Red Flat. ...................... 10

Bloomsdale Extra Early Red Flat................ 15150

Large Red Wethersfield (Landreth's) .............. 10100

" . " " (Ferry's) .............. $10 \quad 85$

Southport Red Globe

Yellow Danvers............................... $10 \quad 75$

Prize Takers (Landreth's) ..................... 15200

White Portugal -

(Ferry's) _............................ is 150

Suuthport White Globe

Mammoth Silver King ........................ 10125

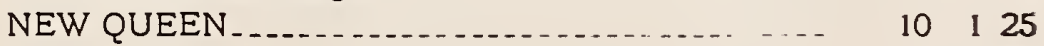

Barletta $\ldots \ldots \ldots \ldots 15175$

Onion Sets-White Bottom $\ldots \ldots \ldots$ QT.

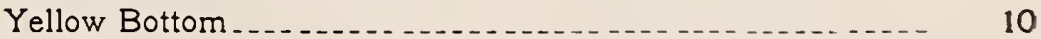

Red Bottom

Red Top Sets .... . . . . 10

White Top Sets ............. 25 
Multipliers $\$ 10$

Winter Sets

Schnitlauch, per clump.

Ask for price per bushel.

Parsley-Champion Moss Curled

Parsnips-Hollow Crown

Bloomsdale

Peas_Landreth's Extra Early (in sealed red hags only)

Ferry's First and Best................... 10

American Wonder

McLean's Little Gem

NOTT'S EXCELSIOR

Alaska. Earliest of all

Everbearing

Advancer

STRATEGEM

Yorkshire Hero

Heroine

Champion of England

Marrowfat Peas.

Field Peas ( $75 \mathrm{c}$ per bushel)

Edible Pod.

Melting Sugar.

If to go by Mail add $8 \mathrm{c}$ per pint.

Pepper_Large Bell Bullnose . . . . . . . . . . . . . $\$ 20$

Ruby King ............................ 20

Golden Dawn . . . . . . . . . . . . . . . . 20

Pumpkin-Common Field...................... 525

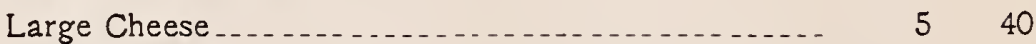

Sweet Sugar ............................... 55

Radishes-Extra Early Scarlet Turnip, White Tip Forcing- $10 \quad 60$

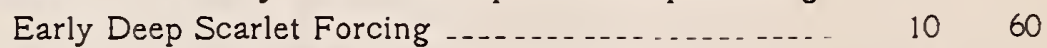

EARLY SCARLET TURNIP WHITE TIP ........ 540

Early Scarlet Turnip ......................... 540

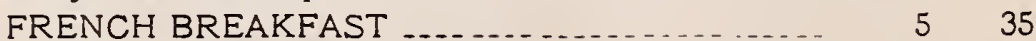

LONG SCARLET SHORT TOP ............... 54

Chartier .............................. 540 
WONDERFUL HALF LONG OR STARTLE(L'd'th's) $\$ 10 \$ 60$ Long White Vienna ........................... 55

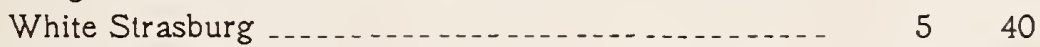

Early White Turnip _............................ 53

Golden Globe

California Mammoth White Winter............... 56

Rose China Winter........................... 560

Black Spanish, Round or Long .................. 55

Salsify-Mammoth Sandwich Island ............... 10100

Spinach-Bloomsdale (Landreth's) _................. 530

Ever Ready ..................................... 530

Long Standing _............................... 55

Squash-Early White Bush (patty pan)

Golden Summer Crookneck..................... 540

HUBBARD, extra warty _......................... 55

Hubbard ...................................... 540

Marblehead ..................................... 50

Tomato-Atlantic Prize _...

Acme

Trophy ....

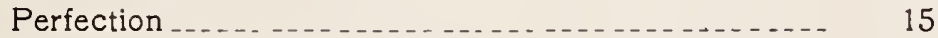

Extra Early Advance.......................... 15

Dwarf Champion.............................. 15

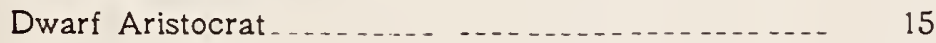

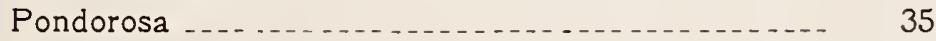

Golded Queen _.................................. 15

Pear Shaped ................................... 20

Ground Cherry _.............................. 25

Crimson Cushion

Turnip-Extra Early White Milan_................... 10100

Extra Early Purple top Milan .................... $10 \quad 75$

Early White Flat Dutch...................... 540

Extra Early Snowball (Landreth's) ............... $15 \quad 75$

Purple Top Strap Leaf ............................. 535

Purple Tọ White Globe....................... 540

Green Top .. “.

Yellow Aberdeen........................... 540

Ruta Baga_Bloomsdale Swede

American Purple Top.......................... 535 


\section{HERB SEED.}

Caraway Seed
Coriander
Dill
Sage
Savory
Thyme
Sweet Marjoram

\section{FLOWER SEED.}

Our aim and ambition is to furnish the best Flower Seed that can be had, from the most reliable growers in the world We quote the leading varieties as follows:

Asters, mixed sorts, many colors $1 / 4 \circ z$. $\$ 50$

“. named varieties 60

Balsam, all colors, mixed sorts 15

Balloon Vine 10

Calliopsis

Candytuft 5

Canna, mixed sorts 5

named varieties

$\$ 15$ to 25

Carnations, mixed sorts

named varieties

Chrysanthemum, annual, mixed sorts 75 to 125

Cowslip

Cypress Vine

Dahlia, single, mixed

“. double,

Daisy

Dusty Miller (Candidisima)

100

Forget Me Not

Four O'Clock

Fox Glove

Fuchsia $-5 c$ for 10 seeds.

Geranium

Golden Rod

Gourds

Heliotrope

Hollyhock 
Mignonette

Moonflower.

Morning Glory, tall or dwarf . . . . . . . . . . . . . . . 10

Moss Rose (Portulacca)

Nasturtium, tall sorts $10 \mathrm{c}$ per oz.

dwarf sorts

Nicotiana

Pansies

Petunia, fine mixed

Phlox

Pink, double mixed

Poppy, double Carnation flowered..................

Sensitive Plant. ...

Stocks

$1 / 8$ oz. $50 \mathrm{c}$,

Sweet Peas, fine mixed

oz. $5 \mathrm{c}$.

". named sorts.

oz. 5 to $10 \mathrm{c}$.

Sweet Williams

Verbena, fine mixed

Violets.blue

Wall Flowers

Wild Cucumbers

Zennia

\section{FLOWERING BULBS.}

We offer only selected stock of the best bulbs. Ask for special prices in large quantities.

Amaryllis

EACH. $\$ 15$

Begonias, Tuberose Rooted, single sorts

" " " double ".

Calladium, Elephant's Ear

Canna

Cinnamon Vine

Dahlias

Lilies

Gladiolus

Hyacinths

Lily of the Valey, clumps

Madeira Vine Roots

Resurrection Plant

Tuberoses, White Pearl

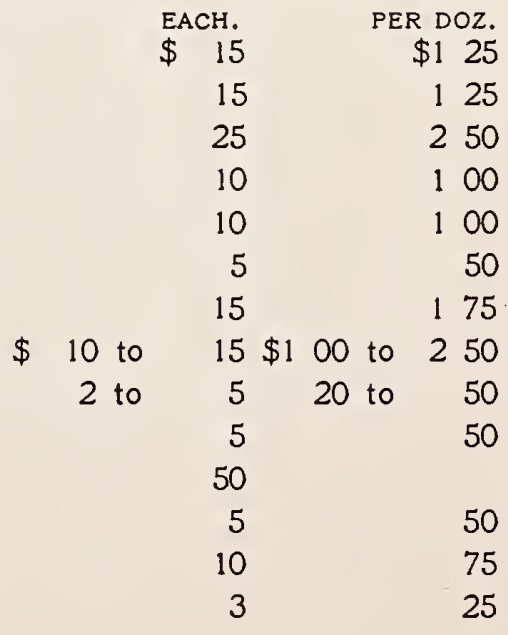




\section{HOME GROWN POTTED BUDDING PLANTS.}

In this we offer only select stock: large size and well grown.

Asters

EACH. DOZ.

Caleus

$\$ 5 \$ 50$

Carnation

10100

Canna

10100

Fuchsia

10100

Dusty Miller

$12 \quad 125$

Heletrope

10100

Geranium, grown in $2 \frac{1}{2}$ inci pots

large, grown in 4-inch pots.............. 35

Marygolds

Petunias

10110

Pansy

5

50

\section{HOME GROWN VEGETABLE PLANTS.}

loose.

We handle all kinds of vegetable plants in season, both potted and

Cabbage Plants, early varieties ready in April _. per doz., 10c. per 100,50c

Late varieties ready in May, same prices. Special in large quantities. Cauliflower, Snowball per doz, 25c

“ potted each, $5 c$

Celery, White Plume - per doz., 10c. per 100,50c

Egg Plant, from select seed per doz., 20c

“ " potted, each, $5 c$

Tomato Plants, early varieties per doz., 10c. per $100,50 \mathrm{c}$ potted, early varieties eacn, 5c. per doz., 50c

\section{GRASS, FIELD AND FARM SEED.}

We quote today's prices on Clover and Grass seed. subject to market changes. Ask for sample and special prices on quantity. Prices here quoted run according to grade and quality. Bags, extra, 15c each.

Remember prices subject to market changes.

Clover-Medium Red.

$\$ 325$ to $\$ 400$ per bu. of $60 \mathrm{lbs}$.

Mammoth

350 to 400

of 60

White

600 to 800 of 60 ..

Alsyke

400 to 500 of 60 .

Alfalfa-Northern grown seed

325 to 375 of $60 \cdots$

Timothy Seed

125 to 150 . of 60 .

Red Top-Fancy Cleaned

10 to $\quad 15$ per $\mathrm{lb}$.

In Chaff 3 to 6 
Blue Grass-Fancy Cleaned Ky.

Extra Cleaned $\mathrm{Ky}$

English Blue Grass

\section{Orchard Grass}

Lawn Grass-Evergreen Western.....

Bromo-lnermis Grass.

Millet-German

Common

New Siberian German

Hungarian

Buy your Millet early; later on prices will range higher.

Seed Corn-lowa Gold Mine

Pride of the North $\$ 100$ per bu of $56 \mathrm{lbs}$.

Duly's Hybrid, earliest large yellow dent.-

Common Yellow Dent

Champion White Pearl

lowa Pearl, early

Squaw Corn

100

100

75

100

100

100

125

White Flint, early

125

Yellow Flin

Pop Corn, select ears for seed
15 per lb.

of 14
35 to 50 per bu. of 48

45 " of 48 of 48 of 48
$2 \mathrm{c}$ per $1 \mathrm{~b}$.

Oats-White Baltic per bu. $\$ 50$

“ Russian ... “ 50

Lincoln

Full Moon _...... . 50

Rape-Essex _...

Common

Broom Corn-Evergreen _._........ “

Buckwheat-Japanese and Silver Hull. Ask for prices in season.

Barley-Beardless ............................ per bu. 100

White Hulless .......... " 100

Common _........... • 35

Rye-Winter, for fodder

True Spring Rye _...

Wheat-Northern Grown Scotch Fife _._. _....... “ 200

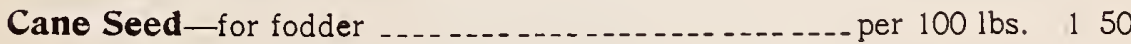

Kaffir Corn-White or Red 


\section{GRAIN BAGS.}

American "A," Seamless Bags

Starks "A," Seamless Bag.

Burlap Ironclad

" Common

SEED POTATOES, FANCY STOCK.

Early Ohio, Red River Grown ...........

" Six Weeks, Red River Grown

Rural N. Y. No. 2 ............ " 125

FERTILIZERS.

Bone Meal per $100 \mathrm{lbs} . \$ 275$

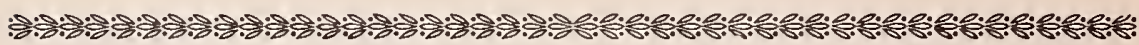
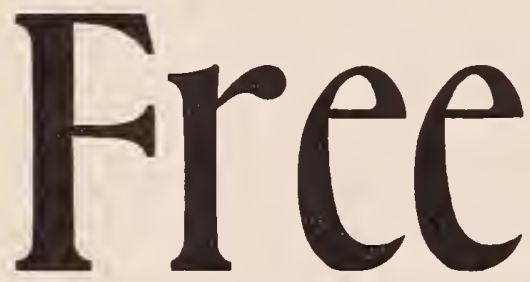

Drop us a postal card, and we will mail you free a catalogue with samples attached and you can select your own suit without coming to Sioux City at lower prices than any clothing house in the West.

\section{Iowa}

Cor.

Fourth and Pearl Sts.
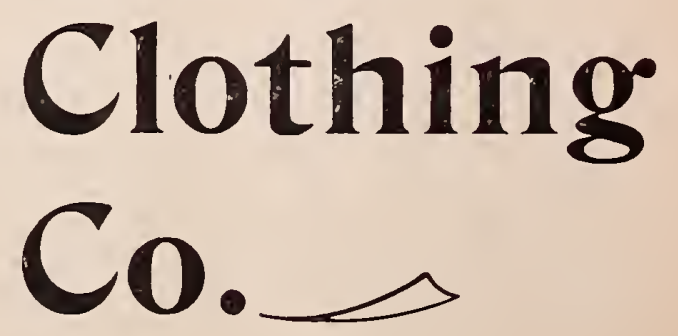


\section{Do You Need A Rest?}

If so stop cultivating with an old hoe and buy one of these PLANET JR. GARDEN TOOLS.

Retails for $\$ 9.00$. My price, Cash $\$ 6.00$.

It's New
A Great
Improve-
ment.
It will
Plant in
Hills or
Continu-
ously.

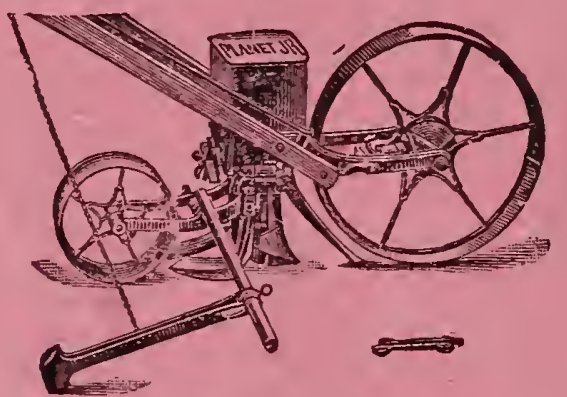

Prices

subject to

Stock

Being

Sold.

I Carry

Full Line

Pepairs.

Single Wheel Hill Dropping Drill.

Retails for \$9.00. My price, Cash, 6.50 .

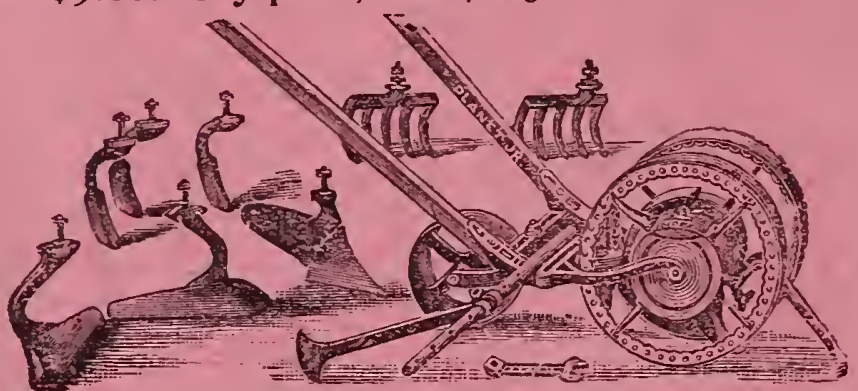

The Plandt Jr., Combined Drill, Wheel Hoe, Cultivator, Rake and Plow.

Retails for $\$ 6.00$. My price, Cash $\$ 5.00$.

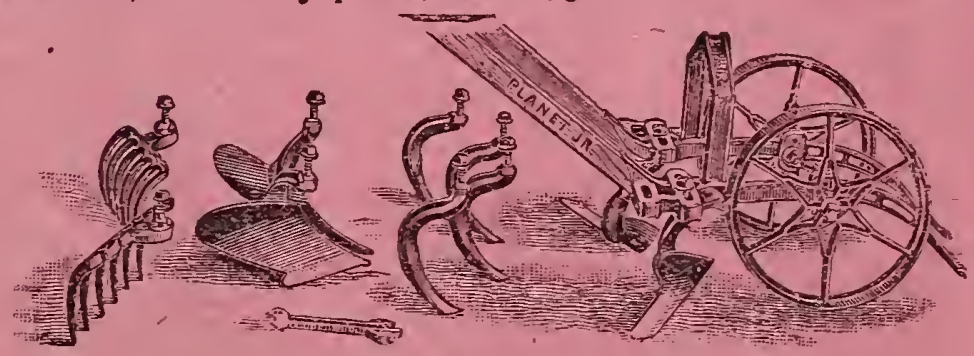

Double Wheel Hoe-Complete.

This does everything except planting.

Fertilizer and seed Drill Combined

Double Wheel Hoe, plain ... 350

Single "*

" " "Fire Fly

Fire Fly Plow a

Eagle Claw Handy Cultivator. 


\section{Michael's Seed Store.}

316 Pearl Street,

SIOUX CITY, IOWA.
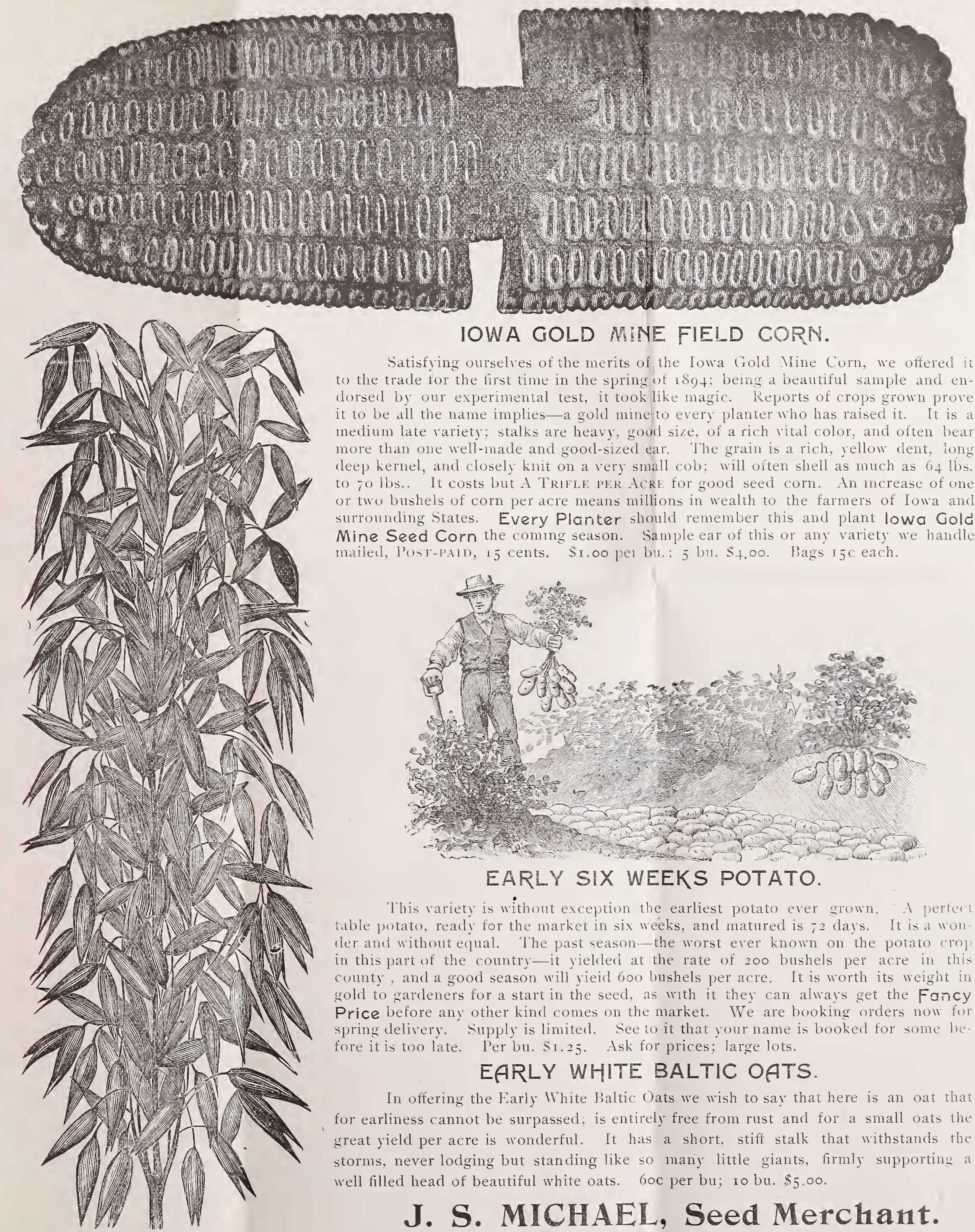

IOWA GOLD MINE FIELD CORN.

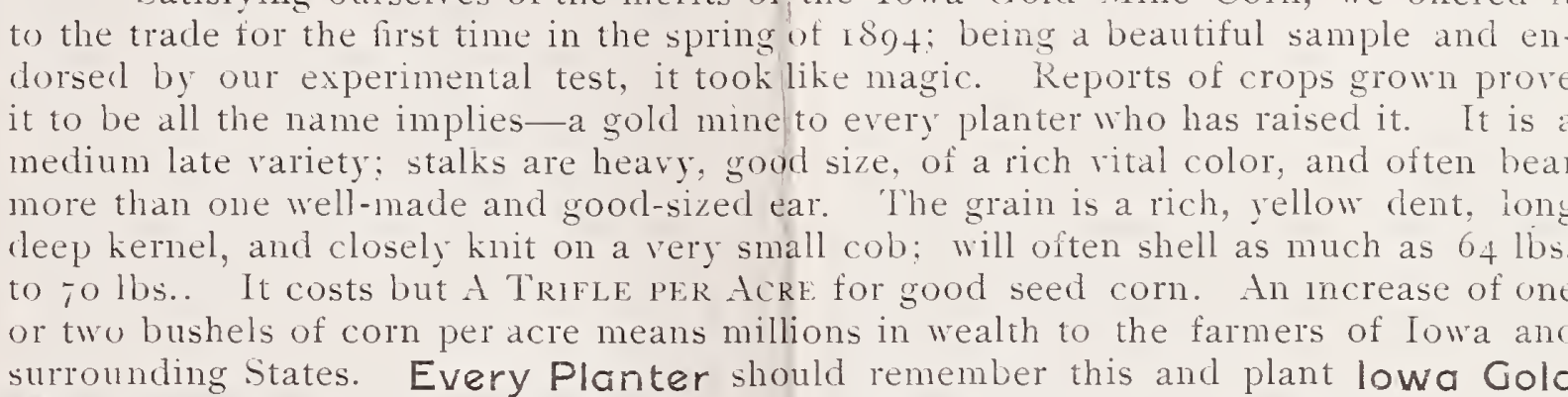
Mine Seed Corn the coming season. Sample ear of this or any variety we handle mailed, Post-pAid, 15 cents. \$r.00 per bu. 5 bu. $\$ 4.00$. Bags i 5 c each.

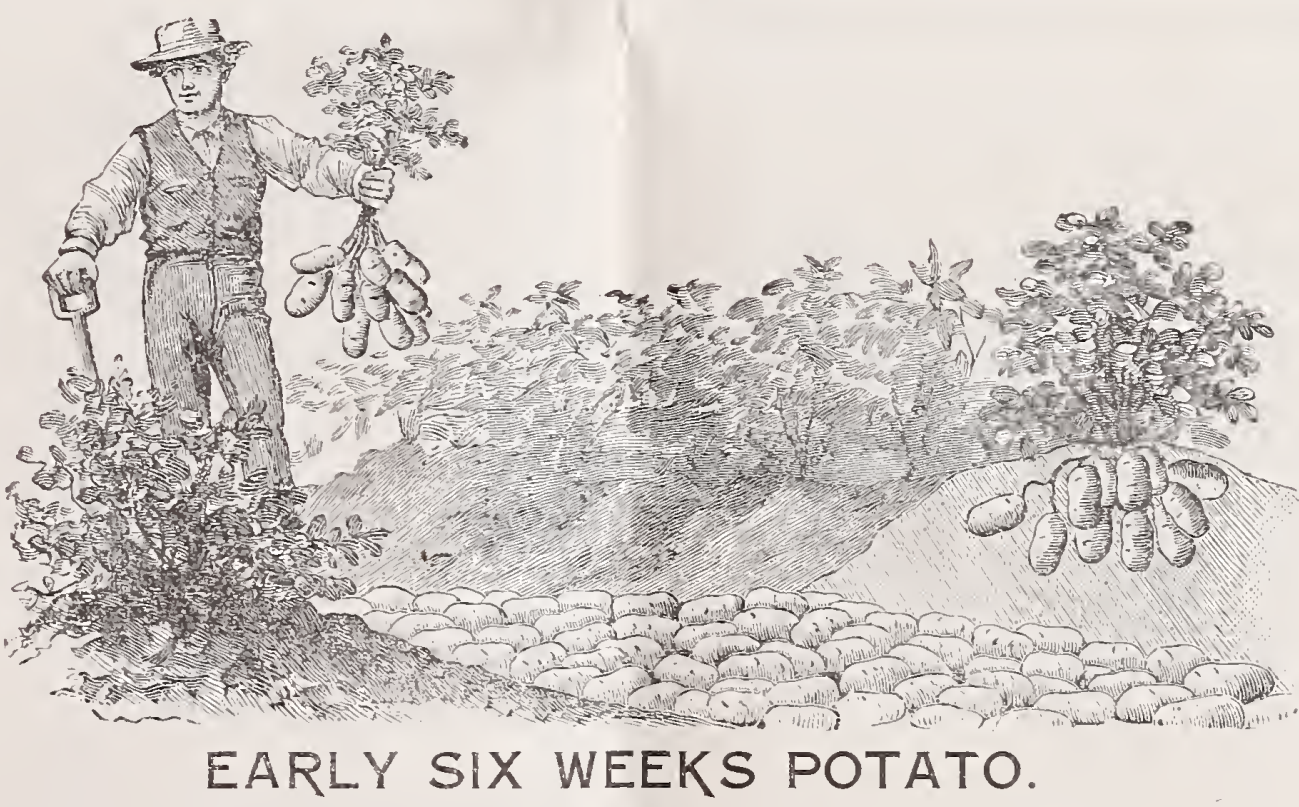

This rariety is withont exception the earliest potato erer grown.

der and without equal. The past season-the worst ever known

in this part of the country-it yielded at the rate of 200 bushels per acre in thi. gold to gardeners for a start in the seed, as with it they can always get the Fancy Price before any other kind comes on the market. We are booking orders now for spring delivery. Supply is limited. See to it that your name is booked for some be fore it is too late. Per bu. \$I.25. Ask for prices; large lots.

\section{EARLY WHITE BALTIC OATS}

In offering the Farly White Baltic Oats we wish to say that here is an oat that for earliness cannot be surpassed; is entirely free from rust and for a small oats the great yield per acre is wonderful. It has a short, stiff stalk that withstands the storms, never lodging but standing like so many little giants, firmly supporting well filled head of beautiful white oats. $60 \mathrm{c}$ per bu; Io bu. $\$ 5.00$. 


\section{Michael's Seed Store.}

\section{SILVER BEARDLESS BARLEY.}

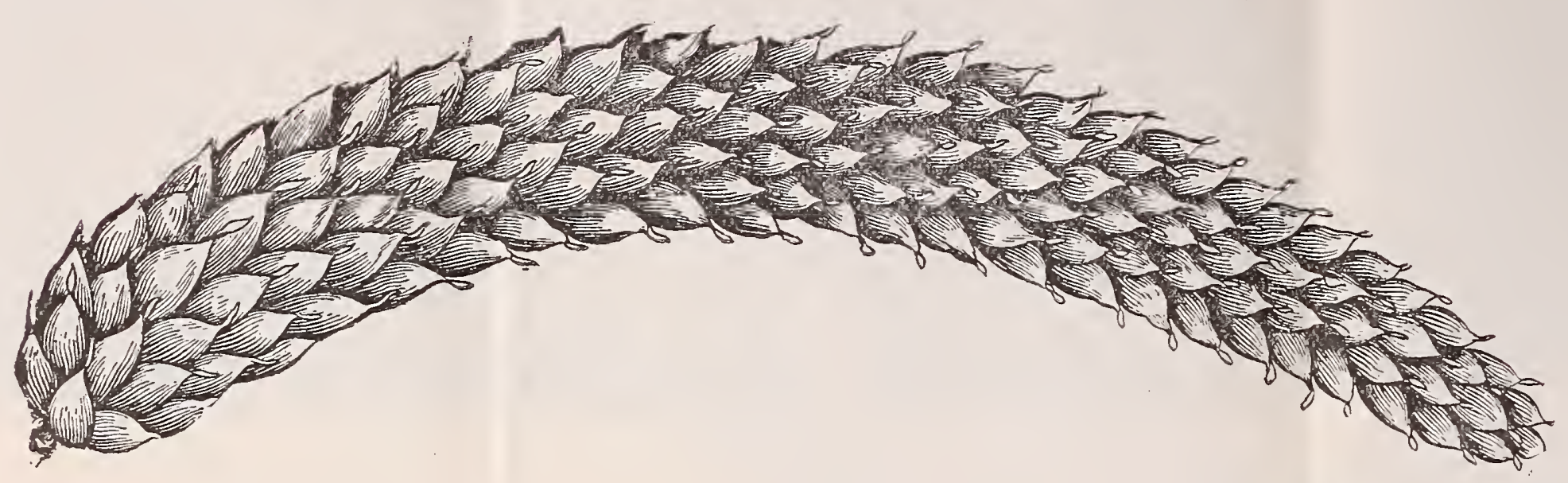

Do you raise barley? If you do then you know how the prickley beards stick you in the harvest. Here is a barley my far. mer friend that overcomes that awful annoyance. A barley that is actually without beards. Just as smooth as wheat. Looks same as any ordinary white barley when threshed; is a wonderful heavy yielder, outyielding the old prickley bearded sorts two bushels to one. Has a stiff, strong straw; stands up well and does not shell out when ripe. Never known to smut, a great stooler; is healthy, strong and vigorous. It is six rowed and has a longer head than most any variety known; is early ripeningwo weeks ahead of the other sorts, in fact possesses every good feature that anybody could wish in a barley. Samples free on application. $\$ 1.00$ per bu.; 5 bu. $\$ 4.00$, ro bu. $\$ 7.50$. Bags extra I $_{5} \mathrm{c}$ each.

\section{DULY'S HYBRID SEED CORN.}
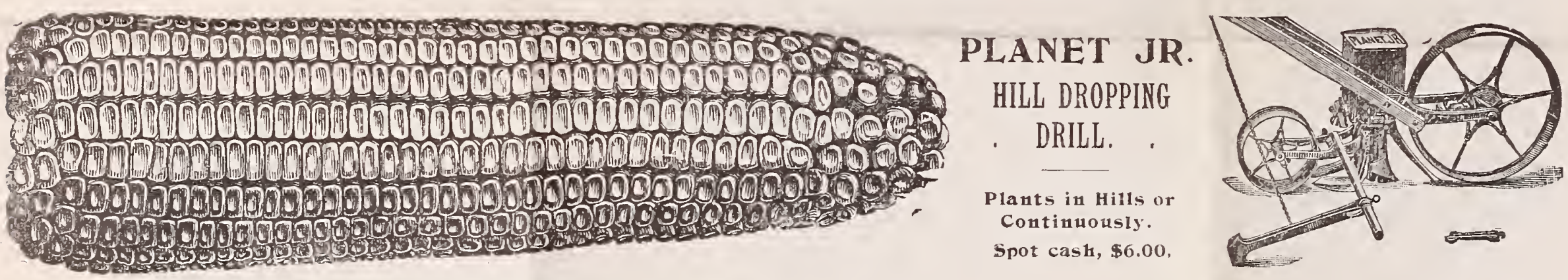

This corn, which we have lately introduced, is a selection we have made from the earliest big yellow dent sorts, now so famous in the upper Missouri Valley country and is the earliest large-eared yellow dent corn known. We introduced it last year. It is a big yielder, nearly as early as the "Pride of the North," and outyields that well-known sort two to one. Fields of this corn planted in June last produced a splendid crop long before September frost came. This is just the corn for the Daota, Iowa and Minnesota farmer, and no planter should fail to plant at least a part of his crop with this excelsior of all early varieties. Samples free. Per bu. \$1.00; 5 bu. \$4.50, ro bu. \$8.50; bags extra I5c each.

\section{ALFALFA CLOVER.}

This valuable forage plant having been tested and tried in the climate and soil of the Northwest with splendid results is now a standard grass crop for this entire region. With few exceptions it has given the best of satisfaction. It should be sown on well cultivated land in the spring as soon as the gronnd is in a tillable condition. Can be drilled or sown broadcast. If drilled $\mathrm{I} 5$ to $20 \mathrm{lbs}$ per acre is sufficient; when sown broadcast 20 to $25 \mathrm{lbs}$ per acre is required. When drilled it should be covered lightly and then cross-harrowed, which insures an even stand. If broadcast a light harrow will cover it well enough. A better stand is always gotten by rolling the land after sowing. It is best to sow it by itself, though on clay or cloddy soil or land given to weeds it is thought best by many to sow a very light sprinkle of wheat, barley or oats as a nurse crop. Some farmers cut it for hay the first season, but this depends entirely upon the rapidity of the growth and is not recommended until the second year. If the field gets weedy the weeds must be mowed and raked off or they will choke out the young plants Once it gets started the Alfalfa soon takes possession of the ground and kills out the weeds. A light sprinkle of manure is of great value for the first winter. It has a wonderful growth and turns as much as three crops per season. The hay is rich and nutritious and valuable feed for all farm animals. It is very valuable as a hog pasture and one acre will keep to to 20 hogs through a season. Every farmer should have a field of Alfalfa. The seed costs but a trifle compared with results. Special prices with samples on application. 